DOI 10. 18307/2017. 0321

(c) 2017 by Journal of Lake Sciences

\title{
新疆巴音布鲁克湿地沉积摇蚊记录对气候变化的响应
}

\author{
宁栋梁 $^{1,2}$, 张恩楼 $^{1 * *}$, 高 光 $^{1}$, 汤祥明 ${ }^{1}$, 郡克强 ${ }^{1}$ \\ (1: 中国科学院南京地理与湖泊研究所湖泊与环境国家重点实验室,南京 210008) \\ (2:中国科学院大学, 北京 100049)
}

\begin{abstract}
摘 要: 基于新疆巴音布鲁克高寒湿地中一碟形洼地沉积岩芯 ${ }^{210} \mathrm{~Pb}$ 测年、摇蚊、沉积理化指标分析结果, 利用冗余分析, 结合气象资料, 探讨了近 $60 \mathrm{a}$ 来气候变化对摇蚊演替的影响. 摇蚊亚化石组合表明, 从 $1990 \mathrm{~s}$ 开始摇蚊优势种从适应性较 强的 Chironomus plumosus-type 向与水生植物关系密切的Dicrotendips nervosus-type、Paratanytarsus penicillatus-type 转变. 几余 分析结果表明总有机碳含量和粒度是影响摇蚊组合演替的主要环境因子,两者共同解释了摇蚊组合变化的 $31 \%$.气候变 化通过改变湿地水量平衡影响水生植被、土壤侵蚀和水体扰动,进而影响摇蚊种群演替.

关键词: 摇蚊组合;底栖生境;气候变化;呪余分析;巴音布鲁克湿地
\end{abstract}

\section{Succession of sedimentary chironomid assemblages in recent years responding to climate change in Bayinbuluk alpine wetland, Xinjiang}

\author{
NING Dongliang ${ }^{1,2}$, ZHANG Enlou $^{1 * *}$, GAO Guang ${ }^{1}$, TANG Xiangming $^{1} \&$ SHAO Keqiang ${ }^{1}$ \\ (1: State Key Laboratory of Lake Science and Environment, Nanjing Institute of Geography and Limnology, Chinese Academy \\ of Sciences, Nanjing 210008, P.R. China) \\ (2: University of Chinese Academy of Sciences, Beijing 100049, P.R.China)
}

\begin{abstract}
The climate pattern has shifted from the warm-dry to warm-wet conditions in the arid northwestern China since 1980s. Changes in ice melting and precipitation in alpine areas caused by this climatic change can alter wetland hydrological processes, which in turn have an effect on biological habitats and ecosystem functions. Together with local instrumental data, this study investigates the effect of climate change on chironomid succession over the past 60 years using the redundancy analysis (RDA) based on sedimentary chironomid assemblages and sedimentary proxies including ${ }^{210} \mathrm{~Pb}$ dating, total organic carbon ( TOC) contents and particle size, from a sediment core derived from a dish-like depression in the Bayinbuluk alpine wetland. Chironomus plumosus-type was the dominance of chironomid species before 1990s, and replaced by phytophilous including Dicrotendips nervosus-type and Paratanytarsus penicillatus-type after that years. RDA results showed that TOC contents and particle size were the most important variables influencing the chironomid succession. The two factors can explain 31 percent of chironomid variations. Climatic through altering the water balance which affects aquatic vegetation, water turbidity and soil erosion, can affect chironomid succession indirectly.
\end{abstract}

Keywords: Chironomid assemblage; benthic habitats; climate change; redundancy analysis; Bayinbuluk alpine wetland

湿地是地表景观的重要组成部分, 占陆地总面积的 $6.4 \%{ }^{[1]}$, 它们供养着独特的植物群落,并为候鸟、两 栖动物以及无脊椎动物等提供栖息和庇护场所 ${ }^{[2-3]}$, 因而也是重要的生态系统组成之一. 然而, 最近的研究 表明,湿地生态系统功能已经并且正在受到气候变化的影响而逐渐退化 ${ }^{[4-8]}$. 水文参数是控制湿地生态系统 结构和功能的关键因子 ${ }^{[2,5]}$, 气候变化可以引起湿地水量平衡改变, 干旱半干旱地区终年降水量少, 蒸发量 大导致水分成为该地区生态状况的限制因子,因而位于干旱半干旱地区的湿地对气候变化表现更为敏感.

\footnotetext{
* 环境保护部环保公益性项目 (201309041) 资助. 2016-06-13 收稿;2016-09-13 收修改稿. 宁栋梁 (1990 ), 男, 硕士研究生;E-mail:ningdongliang14@ mails.ucas.ac.cn.

** 通信作者;E-mail : elzhang@ niglas.ac.cn.
} 
巴音布鲁克高寒湿地位于我国西北干早区天山中部的山间盆地, 是我国唯一的国家级天鹅保护区, 被 誉为 “天山之肾”, 其生态系统保护和合理利用已被列人中国湿地行动计划优先项目 ${ }^{[9]}$. 巴音布鲁克湿地水 源补给以自然降水和冰雪融水为主, 冰雪融水量约占 $15 \%{ }^{[10]}$. 前期研究结果表明, 随着全球气候变暖, 我国 西北地区自 1980s 后期降水量、冰雪消融量和径流量连续多年增加, 气候开始向暖湿方向转变 ${ }^{[11-12]}$. 然而, 对于巴音布鲁克湿地的研究还主要集中在草地利用方式、生物多样性以及地表水变化等方面 ${ }^{[10,13-15]}$, 对历史 时期气候变化对湿地生态系统影响的研究还相对较少 ${ }^{[13]}$.

摇蚊幼虫是水生无脊椎动物的重要组成之一, 因数量丰富并广泛分布于各种淡水环境、对周围环境变 化极为敏感等特点, 常被用作理想的生物监测工具 (bio-monitoring $)^{[16]}$. 摇蚊幼虫难降解的几丁质头壳能够 在沉积物中得到良好保存, 为利用摇蚊头壳亚化石研究历史时期气候变化对生态系统的影响提供了有效途 径 $^{[17]}$, 已被广泛应用于古环境研究中 ${ }^{[18]}$.

本文拟通过对巴音布鲁克湿地中一碟形洼地沉积钻孔中摇蚊头壳亚化石的研究,揭示摇蚊组合演替历 史, 并结合器测数据, 探讨温度和降水等气候因素对摇蚊属种演替的影响, 为在全球变暖趋势下的湿地生态 系统管理和决策提供依据.

\section{1 研究方法}

\section{1 研究区概况}

巴音布鲁克高寒湿地位于天山中部山间盆地,包括大小尤鲁都斯两个湿地,面积 $1369.84 \mathrm{~km}^{2}$,盆地平均 海拔在 2400 $2600 \mathrm{~m}$ 之间, 周围雪山环绕, 山地海拔 $4000 \sim 5500 \mathrm{~m}$. 湿地气候主要受西风带影响, 年平均气 温 $-4.2^{\circ} \mathrm{C}$, 极端最低气温 $-48.1^{\circ} \mathrm{C}$, 年平均降水仅 $280.5 \mathrm{~mm}$, 积雪日数多达 139.3 天, 平均积雪深度 $12 \mathrm{~cm}$, 为 典型的高寒气候 ${ }^{[10]}$. 本研究中的碟形洼地位于巴音布鲁克湿地的东南缘, 为山前洪积扇间洼地, 其内部大 量生长挺水植物苔草, 植被覆盖度较高. 丰富的冰雪资源为大面积的沼泽草地和洼地形成提供了必要条件, 也使其成为博斯腾湖主要水源——开都河的发源地.

\section{2 样品采集与分析}

2013 年利用重力采样器在碟形洼地中心附近 $\left(42^{\circ} 39^{\prime} 40.52^{\prime \prime} \mathrm{N}, 84^{\circ} 22^{\prime} 54.60^{\prime \prime} \mathrm{E}\right.$, 图 1) 采得 $31 \mathrm{~cm}$ 柱状岩芯 一根, 采样点水深 $0.5 \mathrm{~m}$. 现场对岩芯以 $1 \mathrm{~cm}$ 间隔分样, 样品置于自封袋中带回实验室 $4^{\circ} \mathrm{C}$ 冷藏备用.

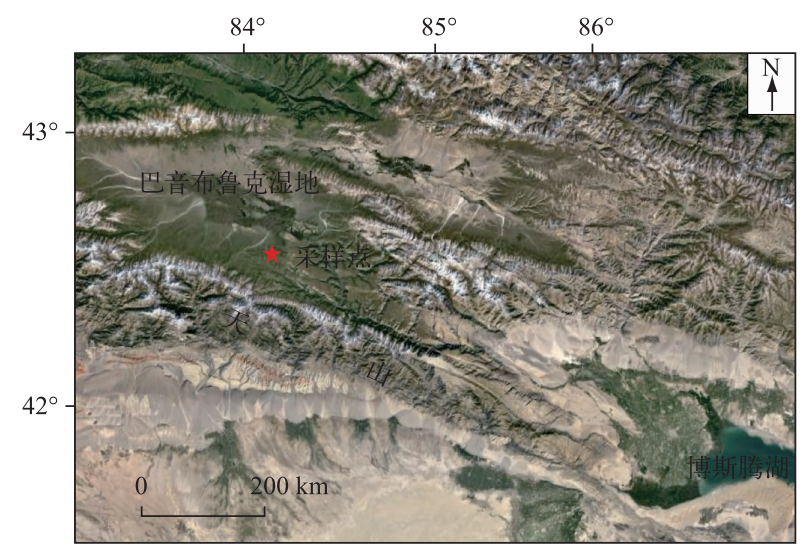

图 1 巴音布鲁克湿地及采样点位置

Fig.1 Location of Bayinbuluk alpine wetland and the sampling site

实验室分析项目包括: ${ }^{210} \mathrm{~Pb}$ 活度、质量磁化率 $\left(\chi_{\mathrm{lf}}\right)$ 、含水量 $(\mathrm{WC})$ 、总有机碳 ( TOC) 含量、粒度和摇蚊. ${ }^{210} \mathrm{~Pb}$ 活度采用高纯锗井型探测器 (HPGe GWL-120-15) 进行测定. 磁化率利用 MS2 磁化率仪进行测定. TOC 含量采用 CE440 元素分析仪测定. 粒度用 Mastersizer 2000 型激光粒度仪测量. 摇蚊样品依据标准方法处 理 ${ }^{[18]}$ : 样品加人 $10 \% \mathrm{KOH}$, 置于 $75^{\circ} \mathrm{C}$ 水浴锅中加热 $15 \mathrm{~min}$ 后依次过 $212 \mu \mathrm{m}$ 和 $90 \mu \mathrm{m}$ 笁, 将剩余样品转移至 
25 倍体视显微镜下, 用铌子将摇蚊头壳手工拣出, 并用 Hydromatrix ${ }^{\circledR}$ 将其封片. 封片后的头壳在 $100 \sim 400$ 倍 生物显微镜下鉴定, 主要依据文献[18-21]进行. 每个沉积样品摇蚊头壳鉴定数量一般不应少于 50 粒 ${ }^{[22]}$, 但 由于本岩芯底部头壳浓度过低, 最少 40 粒摇蚊头壳的鉴定结果依然认为具有代表性 ${ }^{[23]}$. 摇蚊属种百分比图 谱在 TILIA-GRAPH 2.0.b.5 软件 ${ }^{[24]}$ 中绘制, 并基于 CONISS 聚类分析 ${ }^{[25]}$ 划分摇蚊属种组合带.

\section{3 数据统计分析}

降维对应分析 (detrending correspondence analysis, DCA) 用于提取摇蚊种群的动态变化 ${ }^{[26]}$. 在排序分析 中选择至少在两个样品中出现且至少在一个样品中含量超过 $2 \%$ 的属种 (共计 18 个) 参与统计分析. DCA 第 1 轴长度为 1.7 个标准单位 ${ }^{[27]}$, 因此选用线性模式进行分析. 摇蚊幼虫的生长受到多种环境因素的影响, 主要包括温度、水深、食物、底质、 $\mathrm{pH}$ 值、盐度等 ${ }^{[28]}$. 本研究中选取气候指标 (包括温度、降水量和平均风 速 ${ }^{[29]}$ ) 和沉积环境指标 (包括 TOC 含量和中值粒径) 作为解释变量, 以摇蚊组合数据作为响应变量进行冗余 分析 (RDA), 基于蒙特卡洛置换检验 $(P<0.05 ; n=499$; 非限制性置换) 逐步篮选出解释摇蚊演替的显著因 子. 每次选择一个显著因子做解释变量, 余下显著因子做协变量, 进行偏穴余分析 (partial RDA) 计算每个因 子单独作用对摇蚊组合演替的解释份额. 蒙特卡洛置换检验 $(P<0.05 ; n=499$; 非限制性置换 $)$ 用于分析解 释变量的显著水平,所有排序分析用 Canoco 4.5 软件完成 ${ }^{[27]}$.

\section{2 结果}

\section{1 钻孔年代}

本研究中岩芯底部总 ${ }^{210} \mathrm{~Pb}$ 活度未能达到与 ${ }^{226} \mathrm{Ra}$ 的平衡状态 (图 $2 \mathrm{a}$ ), 同时流域侵蚀产沙过程可能影响 $3^{210} \mathrm{~Pb}_{\mathrm{ex}}$ 的来源, 但并未改变其由于衰变而引起的含量随深度增加而减少的趋势, 因此可以采用 ${ }^{210} \mathrm{~Pb}-\mathrm{CIC}$ 模 式 (稳定初始浓度模式) 初步估算沉积年代序列 ${ }^{[00-31]}$, 以方便沉积指标同气象数据进行对比. 岩芯不同深度 对应的年代结果见图 $2 \mathrm{~b}$,据此得出岩芯的平均沉积速率为 $0.43 \mathrm{~cm} / \mathrm{a}$.
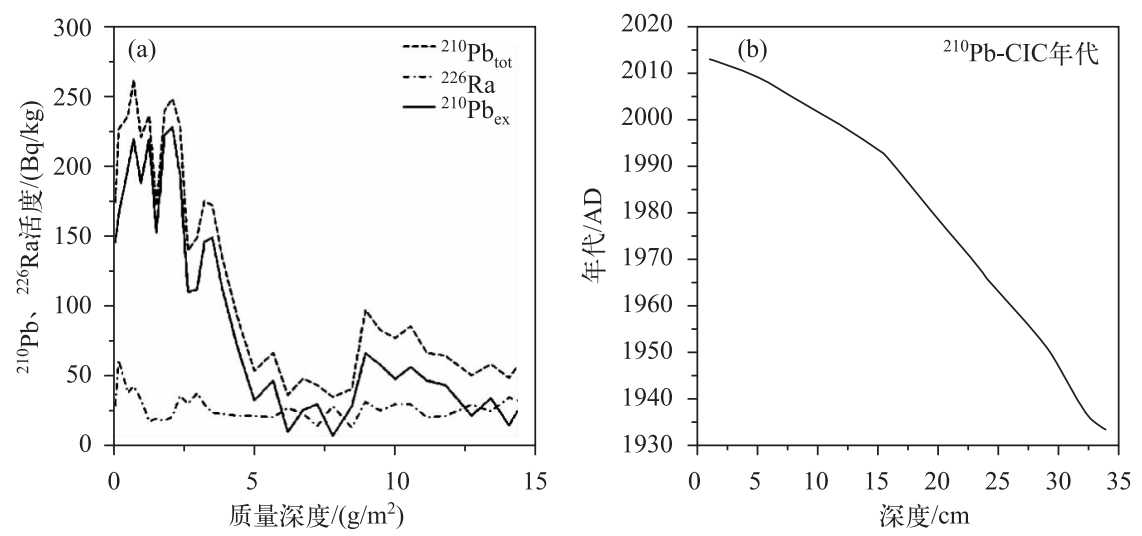

图 2 沉积岩芯 ${ }^{210} \mathrm{~Pb}$ 和 ${ }^{226} \mathrm{Ra}$ 活度随深度的变化 (a); 深度-年代序列 (b)

Fig.2 Variations of ${ }^{210} \mathrm{~Pb}$ and ${ }^{226} \mathrm{Ra}$ activities (a); the sedimentary chronology of the study core (b)

\section{2 沉积物指标变化}

1964 年以前, 沉积物含水量和 TOC 含量较低但呈缓慢增加的趋势, 粒度变化较小, 以细组分 $(<4 \mu \mathrm{m})$ 为主, 中值粒径平均在 $7.5 \mu \mathrm{m}$ 左右; $1960 \mathrm{~s}$ 年到 1990s 中期, 沉积物各项指标变化相对稳定, 仅含水量与磁化 率存在较小的增加趋势; 1990s 中期以来各项指标发生显著变化, 含水量明显增加 (平均 75\%), 磁化率和 TOC 显著减少, 粒度在此阶段呈现波动上升的趋势, 细颗粒物质含量从 $25 \%$ 减少到 $5 \%$, 而较粗组分 ( $>64$ $\mu \mathrm{m}$ ) 含量却呈与之相反的变化趋势, 中值粒径伴随着粗颗粒物质的增加而不断变粗, 平均值向上增加到 17 $\mu \mathrm{m}$ (图 3). 


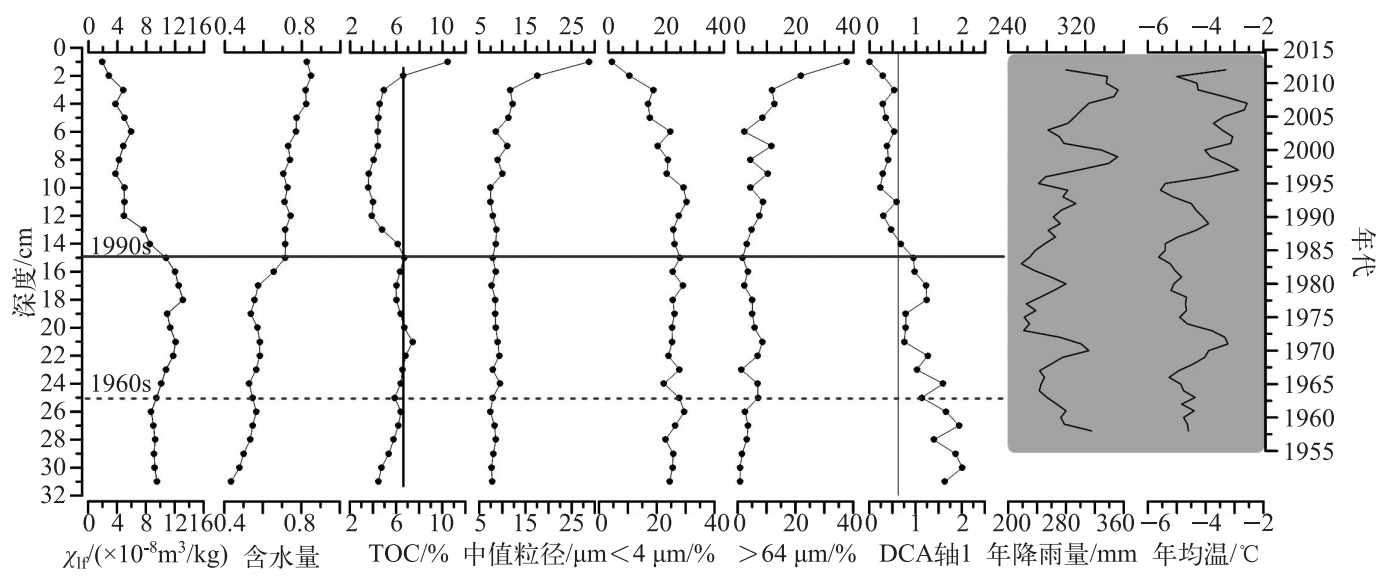

图 3 巴音布鲁克湿地岩芯沉积指标与摇蚊属种 DCA 第 1 轴得分 (DCA axis1) 以及气象数据 （右侧年代轴仅表示气象记录年代,不与左侧深度轴一一对应）

Fig. 3 Sedimentary proxies in the study core, meteorological data of Bayinbuluk alpine wetland and DCA first axis score

\section{3 摇蚊组合演替}

巴音布鲁克湿地钻孔中共鉴定出摇蚊 15 属 18 种, 以与水生植被关系密切的属种为主, 其中, Dicrotendips nervosus-type、Paratanytarsus penicillatus-type 和 Corynoneura edwardsi-type 为主要优势种. Chironomus 相对丰度随深度变浅而逐渐降低, 但在整个钻孔中的含量都相对较高 ( $>12.7 \%$ ), 根据 CONISS 聚类分析,钻孔摇蚊属种可分为两个主要的组合带,其中组合带 I 又可细分为两个亚带 (图 4).

组合带 I a (31 25 cm, 1940s - 1960s): C. plumosus-type 和 C. edwardsi-type 为优势种, 平均含量分别在 40\% 和 20\% 左右, C. edwardsi-type 在该组合带顶部含量快速减少,仅为 7.4\%,D. nervosus-type 在该组合带基 本不出现. 该带摇蚊头壳浓度较低,约为 1 个/g.

组合带 I b $(25 \sim 15 \mathrm{~cm}, 1960 \mathrm{~s}-1990 \mathrm{~s}):$. p plumosus-type 依然是优势种, 但含量呈逐渐减少的趋势, P. penicillatus-type 快速增加并成为优势种, 含量保持在 $15 \%$ 左右, D. nernosus-type 和 Tanytarsus mendax-type 开始出现并呈波动上升的趋势, 但是含量较低 (分别 $10 \%$ 和 3.5\% 左右), C. edwardsi-type 继组合带 I a 顶部 快速减少以后一直保持在相对较低的水平 ( $2.5 \%$ 左右). 该带摇蚊头壳浓度开始逐渐增加, 到组合带 I b 的 顶端已经达到 36 个 $/ \mathrm{g}$.

组合带 II : (15 1 cm, 1990s - 2013 年), D. nernosus-type 含量快速增加, 并与 P. penicillatus-type 一起成 为该带的优势种组合, 含量分别在 $41 \%$ 和 $17 \%$ 左右, C. plumosus-type 逐渐减少, 并在钻孔顶部达到最小值 $(12.7 \%)$, Ablabesmyia monili-type 在该带开始出现, 但含量保持在较低的水平 (3\%左右). 摇蚊头壳浓度快速 上升, 达到整个钻孔的最大值 $(89$ 个 $/ \mathrm{g})$, 平均为 56 个 $/ \mathrm{g}$.

总体而言, 摇蚊组合演替和沉积物各项理化指标变化在时间上较为一致, 特别是 1990s 中期以来 DCA 第 1 轴得分的持续下降与磁化率进一步减小以及含水量、粒度的逐渐增加相对应 (图 3).

\section{4 几余分析结果}

综合上述沉积指标、气候资料数据以及摇蚊数据进行 RDA 分析, 结果表明 (图 5), TOC 含量和中值粒径 $(\mathrm{d}(0.5))$ 代表的粒度是影响近 $60 \mathrm{a}$ 来摇蚊组合演替的主要环境因子, 它们共同解释了摇蚊组合变化的 $31 \%$, 偏冗余分析结果显示, TOC 含量和粒度指标分别单独解释了摇蚊组合方差的 $22 \%$ 和 $8 \%$.

\section{3 讨论}

\section{1 巴音布鲁克湿地气候变化}

巴音布鲁克气象站气象记录 (图 3) 表明, 近 $60 \mathrm{a}$ 来, 流域气候发生较为明显的变化, 降水量呈显著增加 


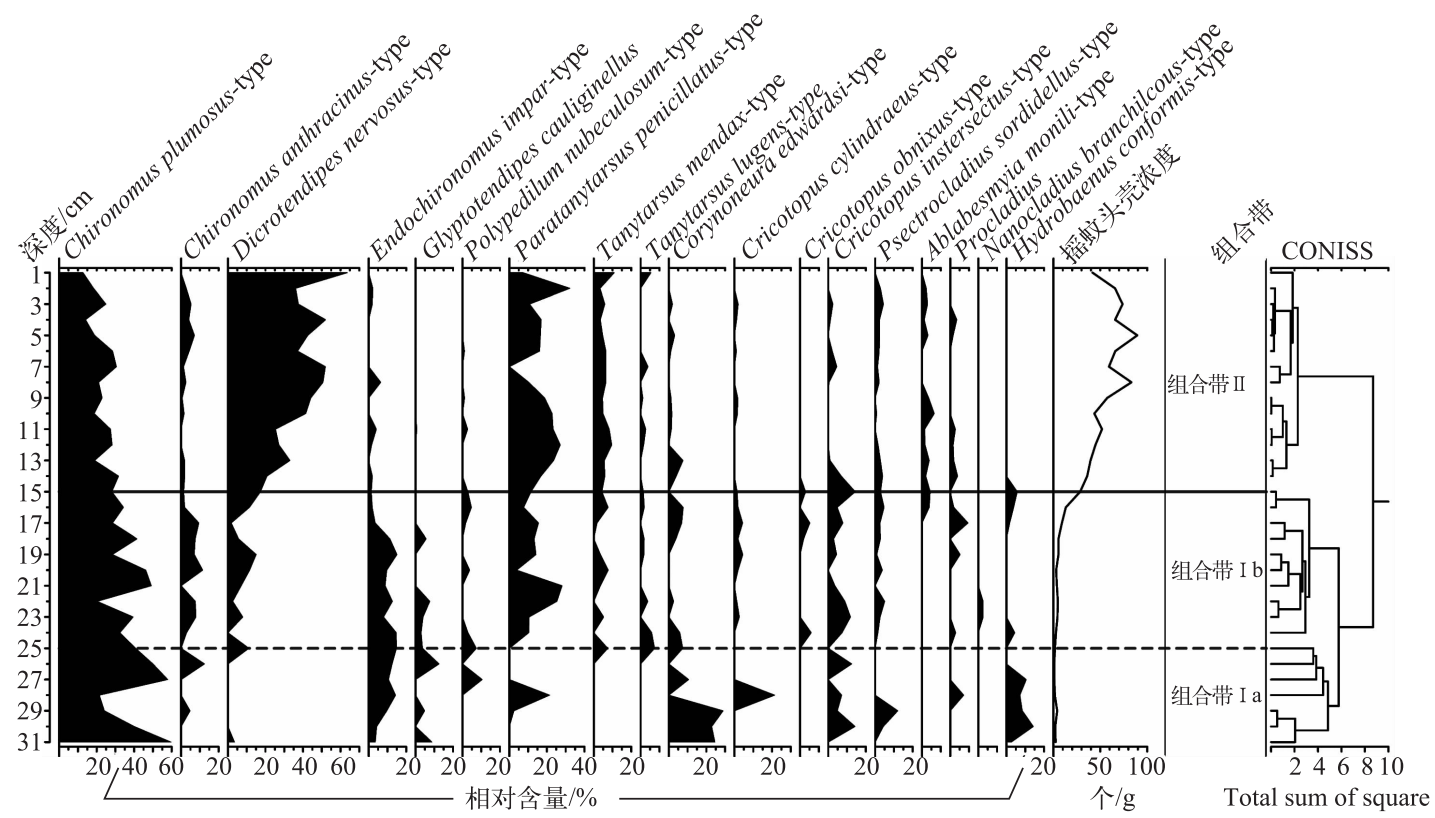

图 4 巴音布鲁克湿地主要摇蚊属种组合图谱以及摇蚊头壳浓度

Fig.4 The main chronomid taxa in percentage abundance (\% ) for Bayinbuluk alpine wetland as well as concentrations of head capsules

的趋势, 而温度也明显升高. 特别是 $1990 \mathrm{~s}$ 以来, 流域年降雨量 平均值 $(301.9 \mathrm{~mm}$ ) 比 1960s 到 1990s 中期 (255.5 mm) 增加了 $46.4 \mathrm{~mm}$,年均温升高 $0.9^{\circ} \mathrm{C}$ ( $1990 \mathrm{~s}$ 中期至 2012 年平均值为 $-4.6^{\circ} \mathrm{C}, 1964$ 年至 $1990 \mathrm{~s}$ 中期为 $-3.7^{\circ} \mathrm{C}$ ), 该记录同西北干旱区 的其他近 $50 \mathrm{a}$ 来的记录一致 ${ }^{[11-12]}$, 都表明该地区气候具有从 暖干向暖湿转变的趋势.

\section{2 摇蚊组合演替对气候变化的响应}

摇蚊头壳浓度于 $25 \mathrm{~cm}(1960 \mathrm{~s})$ 处开始升高, 且 $15 \mathrm{~cm}$ (1990s) 处升高速率迅速增加, 摇蚊组合在相应的时间也发生 了明显改变, 这和岩芯中沉积指标发生变化的时间相一致. 圥 余分析结果表明, TOC 含量和粒度是影响洼地摇蚊组合演替的 主要影响因素 (图 5). 而气候因素 (温度、降水以及风速) 在 RDA 分析中表现并不显著. TOC 含量与 RDA 第 1 轴存在较密 切的负相关关系, 不同深度样点在 RDA 双轴图中划分为两个

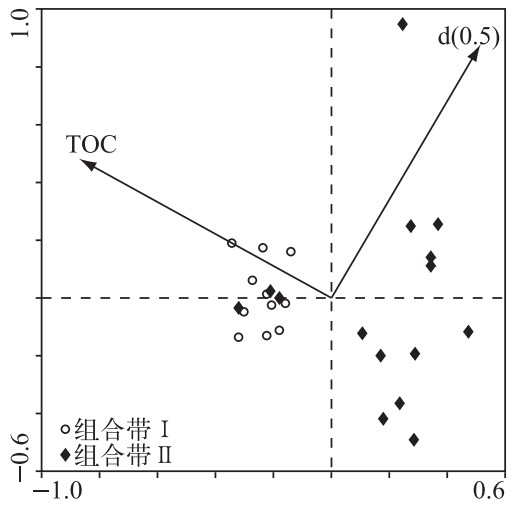

图 5 几余分析双轴图

Fig.5 The biplot of redundancy analysis 明显的组合单元, 两个单元基本与组合带相一致. 气候因素对 生物的影响可能在区域范围内更为明显, 例如 Walker ${ }^{[32]}$ 认为温度是控制摇蚊在大的地理尺度上分布的最为 重要的潜在环境因子,而在地区范围甚至单一水体内部, 气候变化导致的水文理化性质 (如水深、 $\mathrm{pH}$ 值、盐 度和营养状况等) 的改变可能对水生生物的影响更为直接 ${ }^{[33-36]}$. 底栖无脊椎动物生长的底质条件是影响生 物生长和发育的重要影响因素 ${ }^{[26]}$. TOC 含量和粒度是表征底质状况的重要环境指标. 研究结果表明, 细颗 粒沉积物增加可以阻塞底质沉积物内部空隙, 减少底栖生境的含氧量, 改变生物化学过程以及微生物活动, 进而对底栖生物生存和生长产生不利影响 ${ }^{[36-37]}$. 一般而言, TOC 含量可以用来指示初级生产力状况. 本研究 中, TOC/TN 比值变化范围为 7.93 12.75, 平均为 10.51 (顶部 $2 \mathrm{~cm}$ 除外), 表明洼地有机质主要来源于水生 
植被 ${ }^{[38-39]}$, 这和洼地内大量生长的苔草密切相关. 水生植被复杂的空间结构可以为摇蚊提供庇护场, 身避其 他生物的捕食 ${ }^{[40]}$, 同时附着在水生植被上的丰富的细菌和藻类等生物为摇蚊提供了食物来源 ${ }^{[41]}$. 因而水生 植被 (种类或数量) 的增加, 可以促进摇蚊属种丰度和多样性的增加.

该研究中碟形洼地摇蚊头壳在 1990s 以前含量较低, 摇蚊属种以环境适应能力较强的 C. plumosus-type 为主要优势属种, 沉积指标 TOC 含量较高, 而粒度以细颗粒物质为主, 表明洼地初级生产力较高, 水动力条 件较弱. 气象数据显示, 1990s 以前巴音布鲁克山区降水量较少, 温度也相对偏低, 这将导致流域内水源补给 相对缺乏. 考虑到洼地水体较浅 (夏季采样水深 $0.5 \mathrm{~m}$ ), 相对缺乏的水源补给会导致洼地在较高蒸发潜力作 用下变的干涸,成为暂时性的水体. 摇蚊生命周期的大部分时间在水体中度过,稳定的水体条件更有益于摇 蚊繁衍和生长 ${ }^{[42]}$, 因此该阶段洼地间歇性淹水环境可能是导致摇蚊头壳浓度极低的原因. 同时, C. plumosus-type 的幼虫体内含有血红蛋白, 可以忍受含氧量较低的甚至缺氧的环境 ${ }^{[43-44]}$, 而细颗粒物质导 致的沉积底质缺氧的环境也可以使该摇蚊种在该阶段成为主要优势属种. 另外, C. plumosus-type 也被广泛 发现于各种暂时性水体当中 ${ }^{[45-46]}$.

摇蚊头壳浓度和 DCA 第 1 轴指示的摇蚊属种组合在 $1990 \mathrm{~s}$ 发生明显变化, 摇蚊头壳含量快速增加, 摇 蚊优势属种由 C. plumosus-type 向与水生植物关系密切的 D. nervosus-type、T. mendax-type 转变, 其他属种如 P. penicillatus-type 、A. monilis-type 以及 $P$. sordidellus-type 含量也逐渐增加. 各种沉积环境指标在该时期也发 生显著改变, 质量磁化率迅速减小, 含水量显著升高, 沉积物粒度逐渐变粗, 而 TOC 含量显著降低甚至低于 岩芯底部水平. 1990s 开始, 该地区降水量和温度都呈波动上升的趋势, 温度升高会导致巴音布鲁克山区冰 雪消融增加, 而巴音布鲁克湿地水源的 $15 \%$ 来自冰雪融水, 因此温度升高会导致湿地的水源补给更为充沛, 加上降水量增加, 导致洼地周边地表径流量增加, 水动力条件增强, 加速流域的土壤侵蚀作用, 进而导致更 多的陆源碎屑物质被搬运沉积在洼地当中, 该推断得到了沉积物粒度逐渐变粗和磁化率逐渐减小的证据支 持 ${ }^{[47]}$. 另外, 水源补给的增加会延长洼地积水时间或增加洼地积水面积, 这将有利于水生植被的大量发 育 ${ }^{[48]}$. 然而 TOC 含量的减少似乎与该推断相悖. 事实上, 1990s 以来, 大量侵蚀来源的陆源碎屑物质的沉积 增加了沉积物中的无机碳含量 (未发表数据), 对 TOC 产生了较为明显的稀释作用. 气候变化导致的水文条 件改变显著改变了摇蚊属种组合, 粒度变粗导致适应砂和粉砂等细粒沉积物的 C. plumosus - type ${ }^{[49]}$ 含量逐渐 减少,而水生植被为与植被关系密切的摇蚊种提供了良好的栖息环境和食物来源, 促进了 D. nervosus-type、 T. mendax-type、P. penicillatus-type 和 A. monilis-type 的大量发育 ${ }^{[17,50]}$. 另外, 值得注意的是, 摇蚊属 (种) Glyptotendipes biwasecundus 和 Cricotopus spp. 与 P. penicillatus-type 在沉积序列中 (特别是在 $1990 \mathrm{~s}$ 前后) 呈相反趋 势变化. 研究表明前两个摇蚊属 (种) 与水体扰动呈明显正相关关系 ${ }^{[51-52]}$, 而 $P$. penicillatus-type 更适应在开 阔水环境中生存 ${ }^{[50]} .1990 \mathrm{~s}$ 以前, 湿地降水量和温度存在多次较大幅度的波动, 但降水量和温度依然相对较 低,加之洼地积水较浅, 剧烈的气候波动可以导致洼地水体扰动较大, 而 1990s 以来, 降水量的持续增加和温 度的逐渐升高导致的洼地积水时间延长和积水面积的扩大可能促进了 P. penicillatus-type 的发育.

\section{4 结论}

总体而言, 巴音布鲁克高寒湿地近 $60 \mathrm{a}$ 来的气候变化改变了湿地的水量平衡, 导致生物栖息环境发生 改变,进而对摇蚊组合演替产生了显著影响. 近 $60 \mathrm{a}$ 来巴音布鲁克地区降水量和温度都呈增加的趋势, 气候 向暖湿方向转变, 这和我国西北地区整体趋势一致. 温度升高加速冰雪消融, 加上降水量增加, 导致湿地水 源补给更为充沛, 地表径流增加, 进而加剧流域土壤侵蚀, 改变了洼地基底粒度组成, 影响了摇蚊的生长和 演替. 同时水源补给增加有利于湿地水生植被的生长, 为摇蚊的繁衍和生长提供了良好的栖息场所和食物 来源, 促进与水生植物关系密切的摇蚊种的发育. 另外, 气候波动导致的洼地水体扰动和水体面积的变化也 是影响摇蚊种属演替的重要因素.

\section{5 参考文献}

[ 1 ] Wang Xianli, Li Xiuzhen. Advances in wetlands' researches. Chinese Journal of Ecology, 1997, 16(1) : 58-62. DOI: 10. 13292/j.1000-4890. 1997.001. [王宪礼, 李秀珍. 湿地的国内外研究进展. 生态学杂志, 1997, 16(1): 58-62.] 
[ 2 ] Dawson TP, Berry PM, Kampa E. Climate change impacts on freshwater wetland habitats. Journal for Nature Conservation, 2003, 11(1) : 25-30. DOI: 10.1078/1617-1381-00031.

[ 3 ] Mitsch WJ, Wu XB. Wetlands and global change. In: Soil management and greenhouse effect. Boca Raton: CRC Lewis Publishers, 1995.

[ 4 ] Brotherton SJ, Joyce CB. Extreme climate events and wet grasslands: Plant traits for ecological resilience. Hydrobiologia, 2015, 750(1) : 229-243. DOI: 10.1007/s10750-014-2129-5.

[ 5 ] Erwin KL. Wetlands and global climate change: The role of wetland restoration in a changing world. Wetlands Ecology and Management, 2008, 17(1) : 71-84. DOI 10.1007/s11273-008-9119-1.

[ 6 ] Thomas CD, CameronA, Green R et al. Extinction risk from climate change. Nature, 2004, 427(6970) : 145-148. DOI: 10.1038 /nature02121.

[ 7 ] Fu Guobin, Li Kerang. Progress in the study on the relationship between global warming and wetland ecological system. Geographical Research, 2001, 20(1) : 120-128. [傅国斌, 李克让. 全球变暖与湿地生态系统的研究进展. 地理研究, $2001,20(1): 120-128$.

[ 8 ] Song Changchun. Influence of global climate change on wetlands. Wetland Science, 2003, 1(2): 122-127. [宋长春. 湿地 生态系统对气候变化的响应. 湿地科学, 2003, 1(2): 122-127.]

[ 9 ] State Forestry Administration ed. Chinese wetland protection motion plan. Beijing: China Forestry Publishing, 2000. [ 国 家林业局. 中国湿地保护行动计划. 北京: 中国林业出版社, 2000.]

[10] Yang Qing, Cui Caixia. Impact of climate change on the surface water in Bayanbuluk Alpine-Cold Wetland in the Tianshan Mountainous. Journal of Glaciology and Geocryology, 2005, 27(3) : 397-403. [杨青, 崔彩霞.气候变化对巴音布鲁克 高寒湿地地表水的影响. 冰川冻土, 2005, 27(3) : 397-403.]

[11] Shi YF, Discussion on the present climate change from warm-dry to warm-wet in northwest China. Quaternary Sciences, $2003,23(2): 152-164$.

[12] Xu CC, Li JX, Zhao J et al. Climate variations in northern Xinjiang of China over the past 50 years under global warming. Quaternary International, 2015, 358: 83-92. DOI:10.1016/j.quaint.2014.10.025.

[13] Liu Yan, Shu Hong, Li Yang et al. Relationship between dynamic change of vegestion cover and climate factors in Bayinbuluk Grassland of Tianshan Mountains. Advances in Climate Change Research, 2006, (4): 173-176. [刘艳, 舒红, 李 杨等. 天山巴音布鲁克草原植被变化及其与气候因子的关系. 气候变化研究进展, 2006, (4) : 173-176.]

[14] Gong Yanming, Hu Yukun, Adeli Maidi et al. Alpine grassland community characteristics at the different stages of degenerating succession in Bayanbulak. Journal of Arid Land Resources and Environment, 2010, 24(6): 149-152. [公延明, 胡 玉昆, 阿德力. 麦地等. 巴音布鲁克高寒草地退化演替阶段植物群落特性研究. 干旱区资源与环境, 2010,24 (6) : 149-152.]

[15] Hu Yukun, Li Kaihui, Wang Xin et al. The biomass of different species communities of Alpine Meadow in Bayinbulak Region. Resouces Science, 2007, 29(3) : 147-151. [胡玉昆, 李凯辉, 王坴等. 巴音布鲁克高寒草甸不同群落类型的生 物量. 资源科学, 2007, 29(3) : 147-151.]

[16] Armitage P, Cranston PS, Pinder LCV. The Chironomidae: Biology and ecology of non-biting midges. Freshwater Science, 1995, 14(3): 611-738.

[17] Langdon PG, Ruiz Z, Wynne S et al. Ecological influences on larval chironomid communities in shallow lakes: Implications for palaeolimnological interpretations. Freshwater Biology, 2010, 55(3) : 531-545. DOI: 10.1111/j. 1365-2427. 2009.02345

[18] Brooks S, Langdon P, Heiri O et al. The identification and use of palaearctic chironomidae in palaeoecology. QRA Technical Guide No. 10. London: Quaternary Research Association, 2007: 276.

[19] Oliver DR, Roussel ME. The insects and arachnids of Canada, part II. The genera of larval midges of Canada. Agriculture Canada, 1983: 263.

[20] Wiederholm T. Chironomidae of the Holarctic region: Keys and diagnoses. Part 1, Larvae. Entomologica Scandinavica, Lund, 1983

[21] Rieradevall M, Brooks SJ. An identification guide to subfossil Tanypodinae larvae (Insecta: Diptera: Chrironomidae) based on cephalic setation. Journal of Paleolimnology, 2001, 25(1) : 81-99.

[22] Quinlan R, Smol JP. Chironomid-based inference models for estimating end-of-summer hypolimnetic oxygen from south- 
central Ontario shield lakes. Freshwater Biology, 2001, 46: 1529-1551.

[23] Wiederholm T, Eriksson L. Subfossil chironomids as evidence of eutrophication in Ekoln Bay, central Sweden. Hydrobiologia, $1979,62(3)$ : 195-208.

[24] Grimm EC. TILIA version 1.11. TILIAGRAPH version 1.18. In: Gear A ed. A users notebook. USA: Illinois State Museum, Springfield, 1991.

[25] Grimm EC. CONISS: A Fortran 77 program for stratigraphically constrained cluster analysis by the method of incremental sum of squares. Computers \& Geosciences, 1987, 13(1): 13-35.

[26] Hill MO, Gauch Jr HG. Detrended correspondence analysis: An improved ordination technique. Vegetatio, 1980, 42(1/ $2 / 3): 47-58$.

[27] Ter Braak CJ, Prentice IC. A theory of gradient analysis. Advances in Ecological Research, 1988, 18: $271-317$.

[28] Cao Yanmin. The spatio-temporal distribution of chironomid subfossil and its environmental indications in typical lakes from the middle and lower reaches of Yangtze River[Dissertation]. Nanjing: Nanjing Institute of Geography and Limnology, Chinese Acedamy of Sciences, 2013. [ 曹艳敏. 长江中下游地区典型湖泊摇蚊亚化石时空分布及环境意义 [学位论 文]. 南京: 中国科学院南京地理与湖泊研究所, 2013.]

[29] Chen X, Yang XD, Dong XH et al. Nutrient dynamics linked to hydrological condition and anthropogenic nutrient loading in Chaohu lake (southeast China). Hydrobiologia, 2011, 661: 223-234. DOI: 10.1007/s10750-010-0526-y.

[30] Zhang Xinbao, Long Yi, Wen Anbang et al. Discussion on applying ${ }^{137} \mathrm{Cs}$ and ${ }^{210} \mathrm{~Pb}_{\mathrm{ex}}$ for lake sediment dating in China. Quaternary Science, 2012, 32(3) : 430-440. [张信宝, 龙翼, 文安邦等. 中国湖泊沉积物 ${ }^{137} \mathrm{Cs}$ 和 ${ }^{210} \mathrm{Pbex}$ 断代的一 些问题. 第四纪研究, $2012,32(3)$ : 430-440.]

[31] Liu Enfeng, Xue Bin, Yang Xiangdong et al. ${ }^{137} \mathrm{Cs}$ and ${ }^{210} \mathrm{~Pb}$ chronology for modern lake sediment: A case study of Chaohu Lake and Taibai Lake. Marine Geology \& Quaternary Geolog, 2009, 29(6) : 89-94. DOI: 10.3724/SP.J.1140.2009. 06089. [ 刘恩峰, 薛滨, 羊向东等. 基于 ${ }^{210} \mathrm{~Pb}$ 与 ${ }^{137} \mathrm{Cs}$ 分布的近代沉积物定年方法一一以巢湖、太白湖为例. 海洋地 质与第四纪地质，2009, 29(6):89-94.]

[32] Walker IR. CHIRONOMID OVERVIEW A2 - Elias, Scott A, in Encyclopedia of Quaternary Science. Oxford: Elsevier, 2007: 360-366.

[33] Zhang EL, Zheng BY, Cao YM et al. Influence of environmental parameters on the distribution of subfossil chironomids in surface sediments of Bosten lake (Xinjiang, China). Journal of Limnology, 2012, 71(2) : 291-298. DOI: 10.4081/jlimnol.2012.e31.

[34] Zhang EL, Cao YM, Langdon P et al. Within-lake variability of subfossil chironomid assemblage in a large, deep subtropical lake ( Lugu Lake, Southwest China). Journal of Limnology, 2013, 72 ( 1 ) : 117-126. DOI: 10. 4081/jlimnol. 2013.e10.

[35] Brooks SJ, Birks HJB. The dynamics of Chironomidae (Insecta: Diptera) assemblages in response to environmental change during the past 700 years on Svalbard. Journal of Paleolimnology, 2004, 31 (4) : 483-498. DOI: 10. 1023/B: JOPL. $0000022547.984 .65 . \mathrm{d} 3$.

[36] Luoto TP, Rantala MR, Galkin A et al. Environmental determinants of chironomid communities in remote northern lakes across the treeline-Implications for climate change assessments. Ecological Indicators, 2016, 61: 991-999. DOI: 10. 1016/j. ecolind.2015.10.057.

[37] Donohue I, Irvine K. Effects of sediment particle size composition on survivorship of benthic invertebrates from Lake Tanganyika, Africa. Archiv Fur Hydrobiologie, 2003, 157(1) : 131-144. DOI: 10.1127/0003-9136/2003/0157-0131.

[38] Meyers PA. Organic geochemical proxies of paleoceanographic, paleolimnologic, and paleoclimatic processes. Organic Geochemistry, 1997, 27(5/6) : 213-250. DOI: 10.1016/S0146-6380(97)00049-1.

[39] Meyers PA. Applications of organic geochemistry to paleolimnological reconstructions: A summary of examples from the Laurentian Great Lakes. Organic Geochemistry, 2003, 34(2) : 261-289. DOI:10.1016/S0146-6380( 02)00168-7.

[40] Becerra-Munoz S, Schramm Jr HL. On the influence of substrate morphology and surface area on phytofauna. Hydrobiologia, 2007, 575(1) : 117-128. DOI:10.1007/s10750-006-0359-x.

[41] Papas P. Effect of macrophytes on aquatic invertebrates: A literature review. Melbourne: Arthur Rylah Institute for Environmental Research, Department of Sustainability and Environment and Melbourne Water, Technical Report Series No. 158, 2007. 
[42] Smol JP, Birks JB, Last WM. Tracking environmental change using lake sediments. Volume 4. Zoological indicators. Dordrecht: Kluwer Academic Publishers, 2003.

[43] Brodersen KP, Quinlan R. Midges as palaeoindicators of lake productivity, eutrophication and hypolimnetic oxygen. Quaternary Science Reviews, 2006, 25 (15) : 1995-2012. DOI: 10.1016/j.quascirev.2005.03.020.

[44] Little J, Smol J. Changes in fossil midge (Chironomidae) assemblages in response to cultural activities in a shallow, polymictic lake. Journal of Paleolimnology, 2000, 23(2) : 207-212. DOI:10.1023/ A: 1008005604602.

[45] Bazzanti M, Seminara M, Baldoni S. Chironomids (Diptera: Chironomidae) from three temporary ponds of different wet phase duration in Central Italy. Journal of Freshwater Ecology, 1997, 12 ( 1 ): 89-99. DOI: 10. 1080/02705060. 1997.9663512.

[46] Bazzanti M, Seminara M, Tamorri C. A note on Chironomids (Diptera) of temporary pools in the National Park of Circeo, Central Italy. Hydrobiological Bulletin, 1989, 23(2) : 189-193. DOI: 10.1007/BF02256736.

[47] Chen Jing'an, Wan Guojiang, Zhang Feng et al. The record of lake sediments in different scales-Taking grain size for example. Science in China: Series D, 2003, 33(6) : 563-568. [ 陈敬安, 万国江, 张峰等. 不同时间尺度下的湖泊沉积 物环境记录一以沉积物粒度为例. 中国科学: D 辑: 地球科学, 2003, 33(6): 563-568.]

[48] Bazzanti M, Grezzi F, Bella VD. Chironomids (Diptera) of temporary and permanent ponds in Central Italy: A neglected invertebrate group in pond ecology and conservation. Journal of Freshwater Ecology, 2008, 23 (2) : 219-229. DOI: 10. 1080/02705060.2008.9664194.

[49] Pinder L. Biology of freshwater Chironomidae. Annual Review of Entomology, 1986, 31(1) : 1-23. DOI: 10.1146/annurev.en.31.010186.000245.

[50] Cao YM, Zhang EL, Cheng GJ. A primary study on relationships between subfossil chironomids and the distribution of aquatic macrophytes in three lowland floodplain lakes, China. Aquatic Ecology, 2014, 48(4) : 481-492. DOI: 10.1007/ s10452-014-9499-7.

[51] Brodersen KP, Odgaard BV, Vestergaard O et al. Chironomid stratigraphy in the shallow and eutrophic Lake Søbygaard, Denmark: Chironomid-macrophyte co-occurrence. Freshwater Biology, 2001, 46 (2) : 253-267. DOI: 10.1046/j. 13652427.2001.00652.x.

[52] Greffard MH, Saulnier-Talbot E, Gregory-Eaves I. Sub-fossil chironomids are significant indicators of turbidity in shallow lakes of northeastern USA. Journal of Paleolimnology, 2012, 47(4) : 561-581. DOI:10.1007/s10933-012-9581-x. 